\title{
The role of pulmonary mesenchymal cells in airway epithelium regeneration during injury repair
}

\author{
Suyun Fang ${ }^{1 \dagger}$, Suhong Zhang ${ }^{1 \dagger}$, Haiting Dai ${ }^{1}$, Xiaoxiang $\mathrm{Hu}^{1,2}$, Changgong $\mathrm{Li}^{3}$ and Yiming Xing ${ }^{1 *}$
}

\begin{abstract}
Background: The airways of mammalian lung are lined with highly specialized cell types that are the target of airborne toxicants and injury. Several epithelial cell types and bone marrow-derived mesenchymal stem cells have been identified to serve as stem cells during injury repair. However, the contributions of endogenous mesenchymal cells to recruitment, expansion or differentiation of stem cells, and repair and reestablishment of the normal composition of airway epithelium following injury have not been addressed.

Methods: The role of mouse pulmonary mesenchymal cells was investigated by lineage tracing using Dermo1-Cre; $R O S A^{m T m G}$ mice. In experimental models of lung injury by lipopolysaccharide and naphthalene, GFP-labeled Dermo $1^{+}$mesenchymal cells were traced during injury repair. In vitro lung explant culture treated with or without lipopolysaccharide was also used to verify in vivo data.

Results: During injury repair, a subgroup of GFP-labeled Dermo $1^{+}$mesenchymal cells were found to contribute to normal repair of the airway epithelium and differentiated into Club cells, ciliated cells, and goblet cells. In Club cellspecific naphthalene injury model, the process of Dermo $1^{+}$stem cell regenerating epithelial cells was dissected. The Dermo $^{+}$stem cells was migrated into the airway epithelium layer sooner after injury, and sequentially differentiated transitionally to epithelial stem cells, such as neuroendocrine cells, and finally to newly differentiated Club cells, ciliated cells, and goblet cells in injury repair.

Conclusion: In this study, a population of Dermo $1^{+}$mesenchymal stem cell was identified to serve as stem cells in airway epithelial cell regeneration during injury repair. The Dermo $1^{+}$mesenchymal stem cell differentiated into epithelial stem cells before reestablishing various epithelial cells. These findings have implications for understanding the regulation of lung repair and the potential for usage of mesenchymal stem cells in therapeutic strategies for lung diseases.
\end{abstract}

Keywords: Lung, Dermo1, Mesenchymal stem cell, Lipopolysaccharide, Naphthalene, Injury repair

\section{Introduction}

Throughout life, multicellular organisms must regenerate cells to maintain the integrity and functions of their tissues after injury, but the capacity to repair the tissue damage may fail due to repeated injury and aging. The adult lung is one of the few organs that has a direct interface with the outside environment. The epithelial cells that line the airways are constantly exposed to

\footnotetext{
* Correspondence: ymxing@cau.edu.cn

† Suyun Fang and Suhong Zhang contributed equally to this work.

${ }^{1}$ State Key Laboratory for Agrobiotechnology, College of Biological Sciences,

China Agricultural University, Beijing, People's Republic of China

Full list of author information is available at the end of the article
}

potential toxic agents and pathogens. Therefore, it must be able to respond quickly and effectively to recover the cellular damage. The cellular hallmark of lung repair after injury is a rapid proliferative and differentiation response ultimately leading to restoration of the airway epithelium. Several origins of the stem cells that repair damaged airway epithelium have been identified [1-4]. However, precise information regarding their emergence and diversification during injury repair is scant.

The conducting airways of the mammalian lung are composed of three major epithelial cell types, namely ciliated cells, non-ciliated Club cells, and neuroendocrine 
(NE) cells. These cells are arranged in an orderly manner to form branches and alveolar structures [5]. The mesenchymal part is crucial in determining the shape and size of the lung, which can be subdivided into sub-mesothelial mesenchyme (marked by the expression of WNT2A and FGF10) and sub-epithelial mesenchyme (marked by the expression of NOGGIN) [6]. Pulmonary mesenchyme contains a host of complex cell lineages including lymphatics, endothelial cells, smooth muscle cells, myofibroblasts, cartilage-forming cells, and mesothelial cells [7]. The normal development of pulmonary mesenchyme is associated with the successful extension and branching of the airway $[8,9]$. Coordination of pulmonary mesenchyme and epithelium is required to form a functional lung.

Various experimentally induced whole lung and airway injury models have been used, such as the toxicant naphthalene (NAPH) mediated abolishing of Club cells and the acute lung injury induced by lipopolysaccharide (LPS). Subsequently, the surviving cells are thought to serve as stem/progenitor cells to restore the epithelium. There are evidences that several cell types serve in this capacity. Fist, a subset of NAPH-resistant variant Club cells has been identified that seems to have the ability to self-renew as well as generate other cell types [3]. Second, the pulmonary neuroendocrine (NE) cells are distinguished mainly by expression of PGP9.5. NE cells reside within a unique microenvironment known as neuroepithelial bodies and undergo expansion subsequent to NAPH injury [10]. In bacterial LPS model, the lung parenchyma is damaged by the generation and release of proteases and reactive oxygen and nitrogen species produced by activated lung macrophages and transmigrated neutrophils in the interstitial and alveolar compartments. The end results are microvascular injury and diffuse alveolar damage with intrapulmonary hemorrhage, edema, and fibrin deposition [11]. Mesenchymal stem cells (MSCs), commonly referred to as adult marrow stromal cells, show capacity to differentiate into a number of mature cell types, including fibroblasts, myofibroblasts, and epithelial cells [12]. Treatment with MSCs protected LPS injured mice from death by decreasing the edema and reducing the inflammatory response [13]. Recent study observed significant number of labeled MSCs had migrated from the bone marrow to the lung lesions and differentiated to macrophages, alveolar epithelial cells, and interstitial fibroblasts and myofibroblasts [14]. As ongoing maintenance of the airways and repair after injury are key to normal respiratory function, precise knowledge of which cell type(s) are recruited to reestablish airway homeostasis and the precise mechanics of how repair is controlled is of significant interest.

Exogenously infused MSCs modulate tissue injury and repair. These properties have led to novel therapeutic strategies involving exogenous administration of MSCs in various injury and disease settings. Despite the broad therapeutic potential of this cell type, the in vivo role of endogenous MSCs remains undefined due to the absence of specific markers. In the current study, we demonstrate that a subgroup of Dermo1 ${ }^{+}$mesenchymal cells serve as MSCs to regenerate airway epithelial cells during LPS and $\mathrm{NAPH}$-induced injury repair in mouse lung. These endogenous MSCs sequentially differentiated transitionally to epithelial stem cells, such as neuroendocrine cells, and finally to newly differentiated Club cells, ciliated cells and goblet cells. Moreover, the Dermo1 ${ }^{+}$MSCs are not $\mathrm{HH} /$ Gli1 signaling regulated mesenchymal cells.

\section{Materials and methods}

\section{Mouse lines}

Dermo1-Cre, Gli1-Cre $e^{E R T 2}$, and Gt (ROSA)26Sor ${ }^{t-}$ $m 4(A C T B-t d T o m a t o,-E G F P) L u o / J$ (referred as ROSA ${ }^{m T m G}$ ) mice were gifts from Parviz Minoo (University of Southern California, USA). Dermo1-Cre; ROSA ${ }^{m T m G}$ and Gli1$C r e^{E R T 2}$; ROSA ${ }^{m T m G}$ mice were generated by crossing Dermo1-Cre and Gli1-Cre $e^{E R T 2}$ with ROSA ${ }^{m T m G}$ mice, respectively. All animals were maintained on a 12-h light/ dark cycle with ad libitum access to water and feed in individually ventilated units in the specific-pathogen-free facility. During the experiment, all procedures, care, and handling of animals were in accordance with the guidelines developed by Beijing Association on Laboratory Animal Care and were approved by China Agricultural University (SKLAB-2015-10).

\section{Tamoxifen administration}

Tamoxifen (Sigma, USA) was dissolved in corn oil (Sigma, USA) at a concentration of $20 \mathrm{mg} / \mathrm{mL}$. For lineage-tracing studies, Gli1-Cre ${ }^{E R T 2}$; ROSA ${ }^{\text {mTmG }}$ mice received five continuous doses of $75 \mathrm{mg} / \mathrm{kg}$ bodyweight tamoxifen via intraperitoneal injection to induce CREmediated GFP expression. Injury was induced after 10 days of chasing.

\section{Injury treatments}

Adult mice (8-12 weeks) were selected for injury with no gender distinction. For LPS injury, $20 \mathrm{~mL} / \mathrm{kg}$ bodyweight avertin (Sigma, USA, $20 \mathrm{mg} / \mathrm{mL}$ ) was intraperitoneally injected to anesthetize the mice. Five milligrams per kilogram bodyweight LPS (Sigma, USA, $1 \mathrm{mg} / \mathrm{mL}$, PBS for control mice) dissolved in PBS (phosphate-buffered saline, $\mathrm{pH}$ 7.4) was intratracheally instilled via a 24gauge venous indwelling needle and a $1-\mathrm{mL}$ syringe. An extra of $0.8 \mathrm{~mL}$ of gas was supplied to flush the liquid uniformly into the more distal bronchioles. Mice woke up naturally and sacrificed at $1,3,5,7$, or 14 days post injury (DPI). For naphathalene injury, $300 \mathrm{mg} / \mathrm{kg}$ bodyweight NAPH (Sigma, USA, $30 \mathrm{mg} / \mathrm{mL}$, corn oil for 
control mice) dissolved in corn oil was intraperitoneally injected. Mice were sacrificed at 1, 3, 5, or 7 DPI. Three to 5 mice were analyzed per injury stage. Each injury process was repeated over three times.

\section{RNA isolation and real-time quantitative polymerase chain reaction (qPCR)}

Tissue RNAs were extracted by Qiagen RNeasy Mini Kit (QIAGEN, Germany) according to the handbook. One microgram of total RNAs was applied to synthesize the first-strand cDNAs by promega M-MLV Reverse Transcriptase (Promega, USA). Primers used for qPCR were designed via Primer3 software. Melting curve and amplification analyses were used to validate the primers. Quantification of targeted genes was performed on Roche LightCycler480 instrument with LightCycler 480 SYBR Green-based real-time qPCR kit reagents (Roche, Switzerland). PCR was conducted using the default thermal cycling parameter setting. Sample expression level was normalized to glyceraldehyde-3-phosphate dehydrogenase $(G A P D H)$ expression.

\section{Tissue collection, fixation, and HE staining}

The lung tissues were collected from mice around 2 months old and fixed in $4 \%$ PFA for $16 \mathrm{~h}$ in $4{ }^{\circ} \mathrm{C}$. For paraffin sections, tissues were dehydrated by gradient ethanol solutions, embedded in paraffin. For frozen sections, tissues were dehydrated by gradient sucrose solutions, embedded in OCT. Tissue sections in $5 \mu \mathrm{m}$ were prepared for hematoxylin-eosin (HE) staining and immunohistochemistry analysis.

\section{Histological analysis}

Lung tissue sections were stained with hematoxylin and eosin for HE analysis. $\alpha$-SMA (A5228, Sigma, US); CC10 (07-623, Meck Millipore, US); CD44 (ab25340), CD90 (ab3105), GFP (ab13970), MUC5AC (ab3649), and SMMHC (ab53219) were from Abcam; DESMIN (sc271677), FOXJ1 (sc-53139), and PDGFA (sc-128) were from Santa Cruz; KI67 (Rm-9106-S0, Thermos Fisher); PGP9.5 (53772, Anaspec); SOX2 (3579) and SOX9 (82630) were from Cell Signaling Technology.

\section{Lung explant culture}

Whole mouse lungs were dissected into small pieces and embedded into growth factor reduced $\mathrm{BD}$ matrigel matrix (BD Biosciences, USA), diluted 1:1 in DMEM/ Ham's F12 medium. After polymerization of the matrigel at $37^{\circ} \mathrm{C}$ in a humidified incubator, lung explants were covered with culture medium (DMEM/Ham's F12, 100 $\mathrm{U} / \mathrm{mL}$ penicillin, $100 \mu \mathrm{g} / \mathrm{mL}$ streptomycin, $10 \% \mathrm{FBS}$ ) with or without $10 \mu \mathrm{g} / \mathrm{mL}$ LPS and cultured at $37{ }^{\circ} \mathrm{C}$ in a humidified incubator under 5\% CO2 for $6 \mathrm{~h}$.

\section{Cell counting and image analysis}

Images were captured by an Olympus BX53 microscope under $\times 20$ objective or Nikon A1 laser scanning confocal microscope under $\times 100$ objective after immunostaining (Additional file 2). By ImageJ software, more than 10 random fields per section under $\times 20$ objective were analyzed for cell quantification.

\section{Statistical analysis}

The experimental data are presented as the mean $\pm \mathrm{SD}$ and were analyzed by paired Student's $t$ test using SPSS21.0 software to compare the difference between samples. $P$ value $<0.05$ was considered significant.

\section{Result}

Dermo $1^{+}$mesenchymal cells modulated regeneration of airway epithelium after LPS injury

The most commonly used experimental model for inducing acute lung injury is the bacterial LPS model. By intratracheal instillation of bacterial LPS, we induced severe pulmonary injury with inflammatory cell infiltration and goblet cell hyperplasia. MUC5AC belongs to the secreted mucin cluster and protects the mucosa from infection and chemical damage [15]. The result of MUC5AC antibody staining and HE staining showed that severe immunopathogenesis was induced at 1 day after LPS injection, and by 14 days, the lung was almost recovered (Fig. 1a). Tnfo encodes an inflammatory cytokine mainly secreted by macrophages. It is involved in the regulation of a wide spectrum of biological processes including cell proliferation, differentiation, and apoptosis [16]. Il6 encodes a cytokine to induce fever after infections produced primarily at sites of acute and chronic inflammation [17]. Tnfo and Il6 were highly expressed on 1 day after LPS injection and gradually decreased to healthy level during injury repair. IL10 is an antiinflammatory cytokine secreted predominantly by monocytes that inhibit secretion of TNF $\alpha$ and IL6 induced by LPS [18]. The expression of Il10 and Muc5ac were also largely increased after injection upon the inflammatory response (Fig. 1b). Various airway and alveoli derived epithelial cells were damaged after LPS challenge, such as Club cells, ciliated cells, and TypeII alveolar cells, the expression of those cell markers, CC10, Foxj1, Spc, and $\mathrm{Ttf1}$, were also reduced respectively on day 1 . In contrast, Pgp 9.5, a marker of NE cells, was dramatically increased on day 1 . By day 14 , along with the cell recovering, the expression of these marker genes was restored as well (Fig. 1c). LPS injury also induced damage of mesenchyme-derived cells, including the condensed mesenchymal cells, the sub-mesothelial mesenchymal cells, and the sub-epithelial mesenchymal cells, as the expression of those cell markers, Dermo1, Wnt2a, and Noggin, were also reduced respectively on day 1 and 

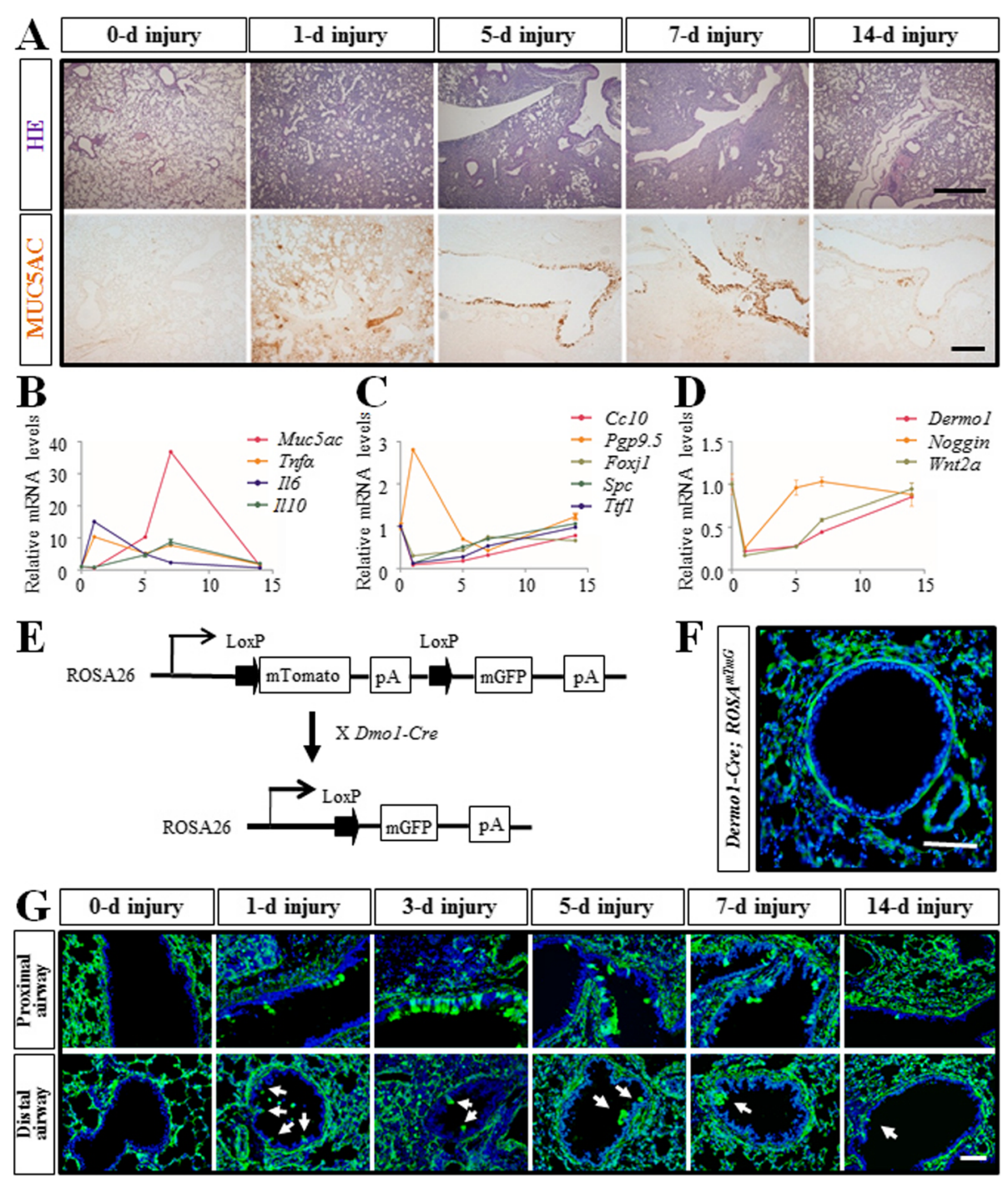

Fig. 1 Generation of LPS injury model and Dermo 1-Cre>; ROSA ${ }^{m T m G}$ mice. a HE staining and MUC5AC immunostaining respectively showed the inflammatory cells infiltration and the goblet cells hyperplasia during the injury-repair process after LPS injury. b Relative mRNA levels of inflammation related genes ( $\mathrm{Tnfa}, 116$, and 1110 ) and mucin gene (Muc5ac) were verified by qPCR. c Relative mRNA levels of different pulmonary epithelial cell marker genes (Cc10, Pgp9.5, Foxj1, Spc, and Ttf1) were verified by qPCR. d Relative mRNA levels of different pulmonary mesenchymal cell marker genes were verified by qPCR. e Schematic of Dermo 1-Cre; ROSA ${ }^{\text {TTmG }}$ mice. $\mathbf{f}$ Immunofluorescence staining showed GFP labeled pulmonary mesenchymal cells in Dermo 1-Cre; ROSA ${ }^{m T m G}$ mice. $\mathbf{g}$ Dermo $1^{+}$lineage cells emerged into the airway epithelium layer after LPS injury. In the proximal airway, GFP ${ }^{+}$cells were clustered while in the distal airway, GFP ${ }^{+}$cells tended to be sporadic (arrow). d, days post LPS injury. Scale bar $=50 \mu \mathrm{m}$

restored on day 14 (Fig. 1d). Thus, by establishing the inflammatory injury model, we simulated the process of the pulmonary injury repair.

Dermo1 is a basic helix-loop-helix transcription factor that is highly expressed in mesodermal tissues in mice [19]. To investigate whether lung endogenous mesenchymal progenitor/stem cells contribute to regeneration of the airway epithelium in injury repair, we have generated Dermo1-Cre; ROSA ${ }^{m T m G}$ mice by crossing Dermo1Cre with ROSA $^{m T m G}$ mice (Fig. 1e) [20]. In Dermo1-Cre;
ROSA $A^{m T m G}$ mice, the Dermo1 positive $\left(\right.$ Dermo $\left.^{+}\right)$mesenchymal cells and all of their progeny express green fluorescent protein (GFP) (Fig. 1f). After LPS injection, Dermo $^{+}$cells were observed on the epithelial layer in both proximal and distal airways on day 1 . The number of Dermo $^{+}$epithelial cells was increased on day 3 and decreased once the injury was repaired (Fig. 1g). The above results suggested that the Dermo1 ${ }^{+}$mesenchymal cells acted as stem cells to regenerate airway epithelium in LPS injury repair. 
Dermo $1^{+}$stem cells proliferated in airway epithelial cell regeneration after LPS injury

As described previously, we identified that a subgroup of Dermo $^{+}$mesenchymal cells acted as stem cells to regenerate airway epithelial cells during LPS injury repair. Therefore, we further examined whether those Dermo $1^{+}$ stem cells went to proliferate once migrated into the airway epithelial layer. By Ki67 antibody staining, proliferating Dermo $1^{+}$epithelial cells were detected as early as $6 \mathrm{~h}$ after LPS injection (Fig. 2a). On days 3 and 5, the proliferating Dermo1 ${ }^{+}$epithelial cells were found mostly aggregating as clusters, and they were hardly detectable on day 14 as the injury was almost repaired (Fig. 2b).

\section{Dermo $1^{+}$stem cells differentiated into various airway epithelial cell types in LPS injury repair}

The major differentiated cellular constituents of the bronchial epithelium include Club cells, ciliated cells, basal cells, goblet cells, and NE cells. To determine which cell types were regenerated by Dermo1 ${ }^{+}$stem cells in LPS injury repair, the expression of cell-specific markers for Club, ciliated, goblet, and NE cells was examined by immunofluorescence. Antibodies to CC10, FOXJ1, MUC5AC, and PGP9.5 were used to double stained with GFP respectively. The overlaid expression pattern of the above markers and GFP indicated that Dermo $^{+}$stem cells were able to give rise to Club cells, ciliated cells, goblet cells, and NE cells (Fig. 3a-d). Distribution of Dermo $1^{+}$ stem cells in differentiating into each cell type was also examined by analyzing the rate of $\mathrm{CC} 10^{\text {positive }}, \mathrm{FOXJ} 1^{\text {positive }}$, MUC5AC ${ }^{\text {positive }}$, and PGP9.5 $5^{\text {positive }}$ cells over total Dermo $^{+}$stem cells. As the largest cell population in airway epithelium, the percentage of $\mathrm{CC}_{10} 0^{\text {positive }} / \mathrm{GFP}^{\text {positive }}$ cells was $93.45 \% \pm 2.52 \%$, which was much higher than the percentages of FOXJ1 $1^{\text {positive }} / \mathrm{GFP}^{\text {positive }}, \mathrm{MUC}^{\mathrm{A}} \mathrm{AC}^{\text {positive }} /$ GFP ${ }^{\text {positive }}$, and PGP9. $5^{\text {positive }} / \mathrm{GFP}^{\text {positive }}$ cells $(6.7 \% \pm$ $4.63 \%, 11.19 \% \pm 15.03 \%$, and $2.56 \% \pm 2.02 \%$, respectively) at 3 days after LPS injury (Fig. 3e).

\section{Dermo $1^{+}$stem cells differentiated into airway epithelial} cells after LPS treatment in vitro

For carefully investigating the movement of Dermo $1^{+}$ stem cells in response to LPS, we performed a lung explant culture experiment. Dermo1-Cre; ROSA ${ }^{m T m G}$ mouse lung explants were dissected and cultured in Matrigel treating with or without LPS. After 6-h treatment, the Dermo1 $1^{+}$cells started to appear in the airway epithelial layer, which was consistent with previous in vivo results (Fig. 4A). Moreover, the Dermo1 ${ }^{+}$cells were proliferating after migrating into the epithelial layer (Fig. 4B). Interestingly, some clusters of Dermo ${ }^{+}$ cells were also observed right under the epithelial cell layer (Fig. 4C (b)). The different locations of Dermo1 ${ }^{+}$ cell clusters around the epithelial cell layer might simulate the movements of Dermo1 $1^{+}$stem cells in LPS injury repair.
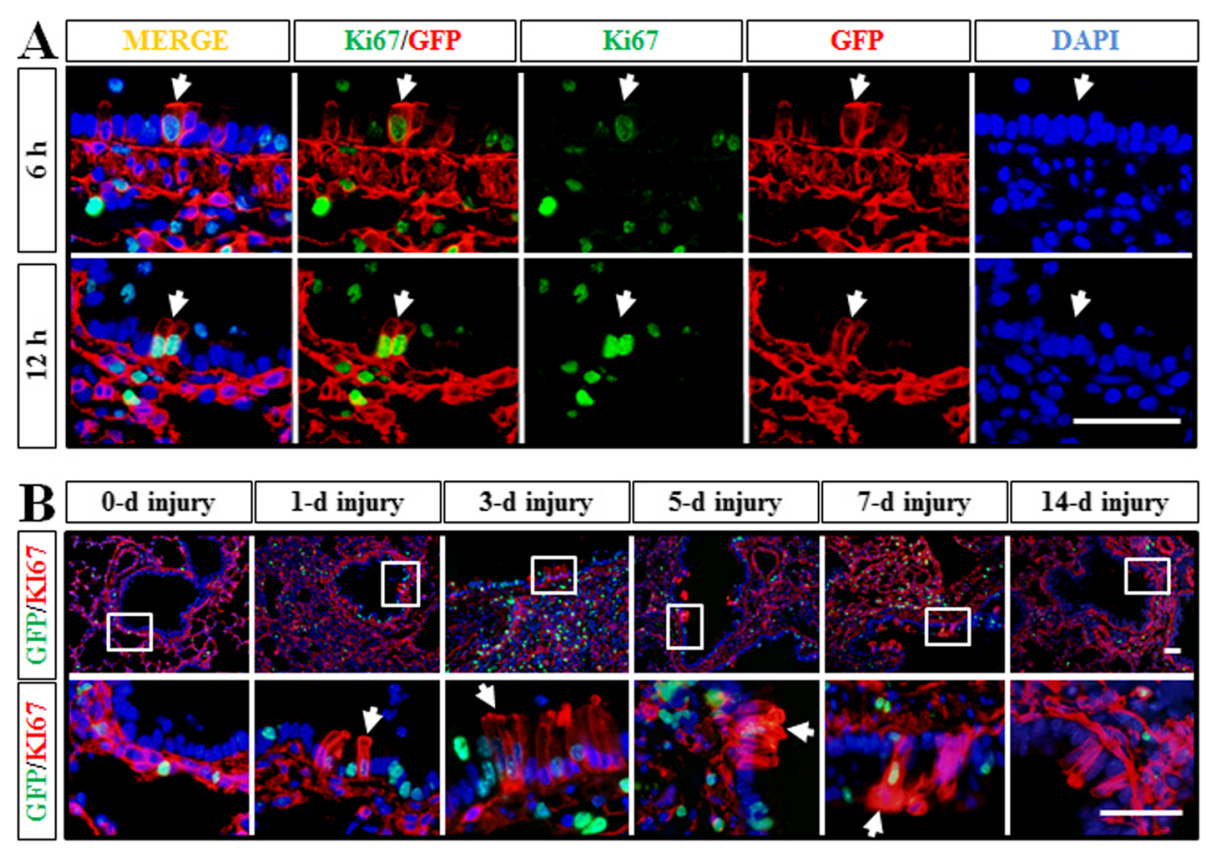

Fig. 2 Proliferation analysis of Dermo $1^{+}$stem cells in LPS injury repair. a By Ki67 antibody staining, proliferating Dermo ${ }^{+}$stem cells were detected right after acute LPS injury at $6 \mathrm{~h}$ and $12 \mathrm{~h}$. $\mathbf{b}$ Dermo ${ }^{+}$stem cells proliferated (arrow) to regenerate the airway epithelium in LPS injury repair. Isolated imagines of enlarged views of $\mathrm{GFP}^{+} \mathrm{Ki} 67^{+}$cells in the airway epithelium were indicated as white boxes. $d$, days post LPS injury. Scale bar $=50 \mu \mathrm{m}$ 

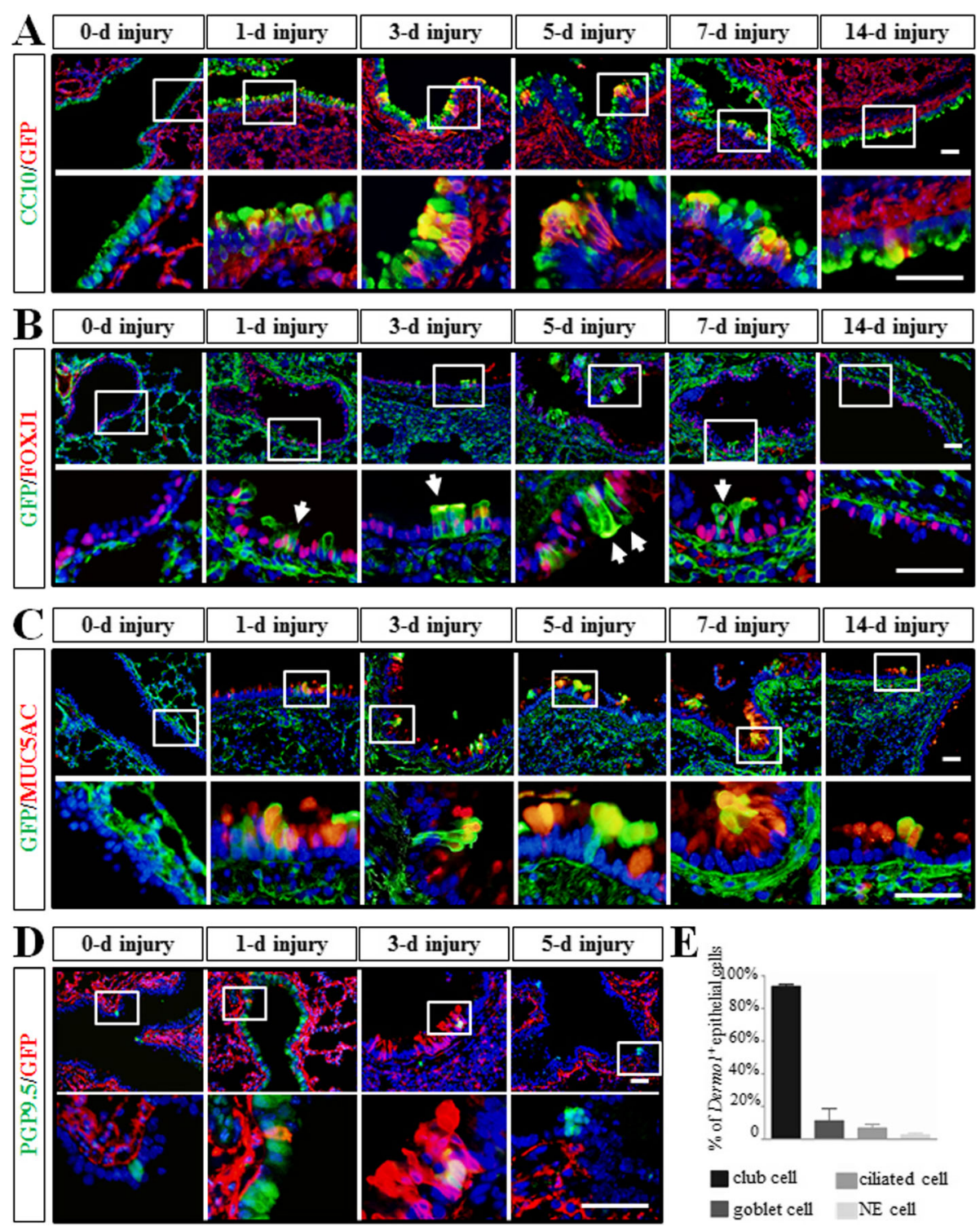

Fig. 3 Dermo $1^{+}$mesenchymal stem cells regenerated diversified airway epithelial cells after LPS injury. Dermo ${ }^{+}$stem cells differentiated into Club cells (a), ciliated cells (b), goblet cells (c), and NE cells (d) after LPS injury. Isolated imagines of enlarged views were indicated as white boxes. e Statistics of Dermo $1^{+}$mesenchyme-derived Club cells, goblet cells, ciliated cells, and NE cells over total Dermo ${ }^{+}$stem cells in the proximal airway at 3 DPI. d, days post LPS injury. Scale bar $=50 \mu \mathrm{m}$

Dermo $1^{+}$stem cells regenerated Club cells through NE cells in NAPH-induced airway injury

There is a significant body of data suggesting that NAPHresistant Club cells and NE cells act as stem cells in airway regeneration after NAPH injury. In our previous work, we proved that NE cells are important source of stem cells to regenerate Club cells in NAPH injury repair [10]. Experimentally induced airway injury by NAPH is a commonly used injury model which abolishes Club cells. Subsequently, the surviving cells are considered to serve as stem cells to restore the bronchiolar epithelium. It deserved attention to identify whether Dermo1 ${ }^{+}$stem cells transdifferentiated into epithelial stem cells, such as NE cells, in Club cell regeneration. Thus, we explored 2-month-old Dermo1-Cre; ROSA ${ }^{m T m G}$ mice to NAPH $(300 \mathrm{mg} / \mathrm{kg}$ body weight). Mazola corn oil was used as negative control. Lung tissues were analyzed on post-NAPH days $1,3,5$, and 7. Antibodies to CC10, PGP9.5, and GFP were used to label Club, NE, and Dermo $1^{+}$cells respectively. Consistent with previous findings, by day 3 after NAPH treatment, the majority of $\mathrm{CC} 10^{\text {positive }}$ cells were killed and released from the airway basement membrane, and the 


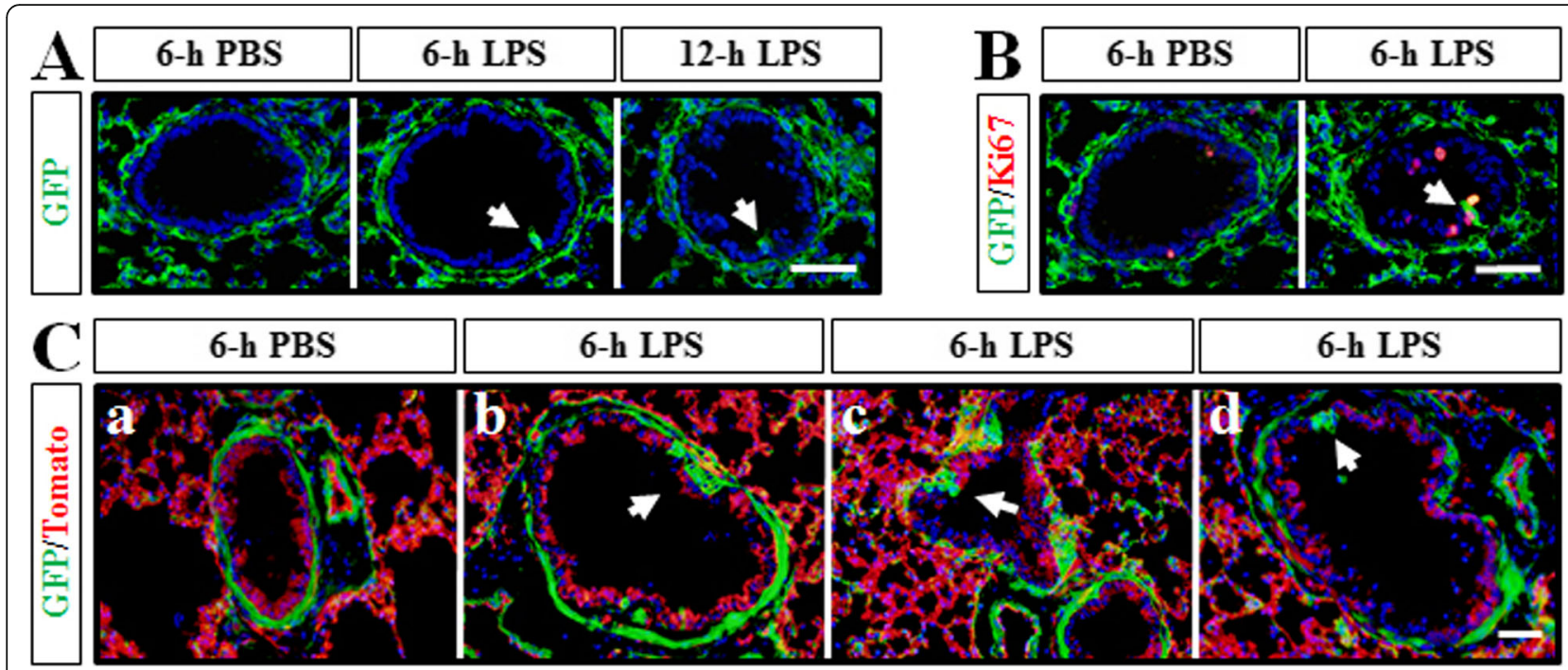

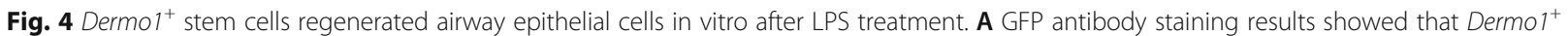
cells emerged into the airway epithelium (arrow) at $6 \mathrm{~h}$ and $12 \mathrm{~h}$ after LPS supplementary in the culture medium. B Ki67 immunostaining results showed that proliferating Dermo ${ }^{+}$stem cells (arrow) were detected in the airway epithelium after $6 \mathrm{~h}$ of LPS treatment. C After 6-h treatment of LPS, Dermo $1^{+}$cells occurred in the airway epithelium with different migration-like forms (arrow) in frozen sections. Scale bar $=50 \mu \mathrm{m}$

airway surface area was covered by PGP9.5 $5^{\text {positive }}$ cells. On day 5 after $\mathrm{NAPH}$ exposure, newly regenerated $\mathrm{CC} 10^{\text {posi- }}$ tive cells comprised the airway epithelium. Subsequently, on day 7 , the Club cell restoration was almost complete and the amount of the PGP9.5 $5^{\text {positive }}$ and $\mathrm{CC} 10^{\text {positive/ }}$ PGP9.5 $5^{\text {positive }}$ cells were significantly reduced (Fig. 5A, G). By double-fluorescence immunostaining of GFP with CC10 or PGP9.5 respectively, we examined the role of Dermol $^{+}$cell in Club cell regeneration. Interestingly, both GFP ${ }^{\text {positive }} /$ PGP9. $5^{\text {positive }}$ and GFP positive $^{\text {CC } 10^{\text {positive }}}$ cells were identified on day 1 after injury, which was earlier than the appearance of PGP9. $5^{\text {positive }} / \mathrm{CC} 10^{\text {positive }}$ (Fig. 5A-D). By analyzing the number of GFPpositive/ PGP9. $5^{\text {positive }}, \mathrm{GFP}^{\text {positive }} / \mathrm{CC} 10^{\text {positive }}$, and $\mathrm{CC} 10^{\text {positive }} /$ PGP9.5 $5^{\text {positive }}$ cells, it confirmed that the presence of GFP ${ }^{\text {positive }} /$ PGP9. $5^{\text {positive }}$ cells started on day 1 and the number was increased on day 3 . Alone with the airway recovery, the population of $\mathrm{GFP}^{\text {positive }} / \mathrm{PGP} 9.5^{\text {positive }}$ cells was reduced on days 5 and 7 (Fig. 5E). Meanwhile, GFP ${ }^{\text {po- }}$ sitive $/ \mathrm{CC} 10^{\text {positive }}$ cells increased since day 1 and peaked at day 7 (Fig. 5). Thus, our analysis of dynamic changes in PGP9. $5^{\text {positive }} /$ Dermo $^{+}$cell population subsequent to $\mathrm{NAPH}$ injury indicated a progression from NE/ Dermo1 $^{+}$cell to NE cell transitional cell intermediates and eventually newly regenerated Club cells. Since NAPH-resistant Club cells and NE cells are stem cells in $\mathrm{CC} 10$ regeneration, the above data suggested that the Dermo1 $^{+}$mesenchymal stem cells differentiated into Dermo1 ${ }^{+}$epithelial stem cells after migrating into epithelial layer. Finally, the Dermo $1^{+}$epithelial stem cells terminally differentiated into new Club cells on days 5 and 7 .

\section{Dermo $1^{+}$stem cells were not Gli1 signaling related}

Recent work on the role of $\mathrm{Gli1}^{+}$pulmonary cells has revealed the importance of SHH-Gli1 in regulating a feedback loop to maintain a balance between proliferation and quiescence during lung homeostasis and regeneration. GLI1 is expressed predominantly in mesenchymal cells adjacent to the proximal airway and pulmonary artery. In our studies, we have found the sub-epithelial mesenchymal layer which was next to the airway epithelium appeared significantly thickened after NAPH injury (Fig. 6a). Therefore, Gli1-Cre ${ }^{E R T 2} ; \operatorname{ROSA}^{\text {mTmG }}$ mice were generated to lineage trace $\mathrm{Gli1}^{+}$cells in adult lung. Induction of CRE-mediated GFP expression labeled Gli1 ${ }^{+}$ cells mainly located surrounding the airway epithelium and vessels. Only a spot of $\mathrm{GFP}^{+}$cells scattered in the sub-mesothelial mesenchyme (Fig. 6b, c). The Gli1$C r e^{E R T 2}$; ROSA ${ }^{m T m G}$ mice were exposed to LPS, and the behavior of $\mathrm{Gli1}^{+}$cells was traced by GFP expression. Surprisingly, the $\mathrm{Gli1}^{+}$cells appeared in the airway epithelium during LPS-induced injury repair (Fig. 6d). Therefore, the SHH-Gli1 signaling was not required for the function of Dermo1 ${ }^{+}$mesenchymal stem cells in epithelium regeneration.

\section{Dermo $1^{+}$stem cells were endogenous mesenchymal stem} cells

A simple plausible cause of the presence of Dermo $1^{+}$ cells in the airway epithelial layer after injury may be bone marrow-derived stem cells through circulating blood. To examine this possibility, we used antibodies to CD44 and CD90, markers of bone marrow-derived mesenchymal stem cells, in Dermo1-Cre; ROSA ${ }^{\text {mTmG }}$ lungs 

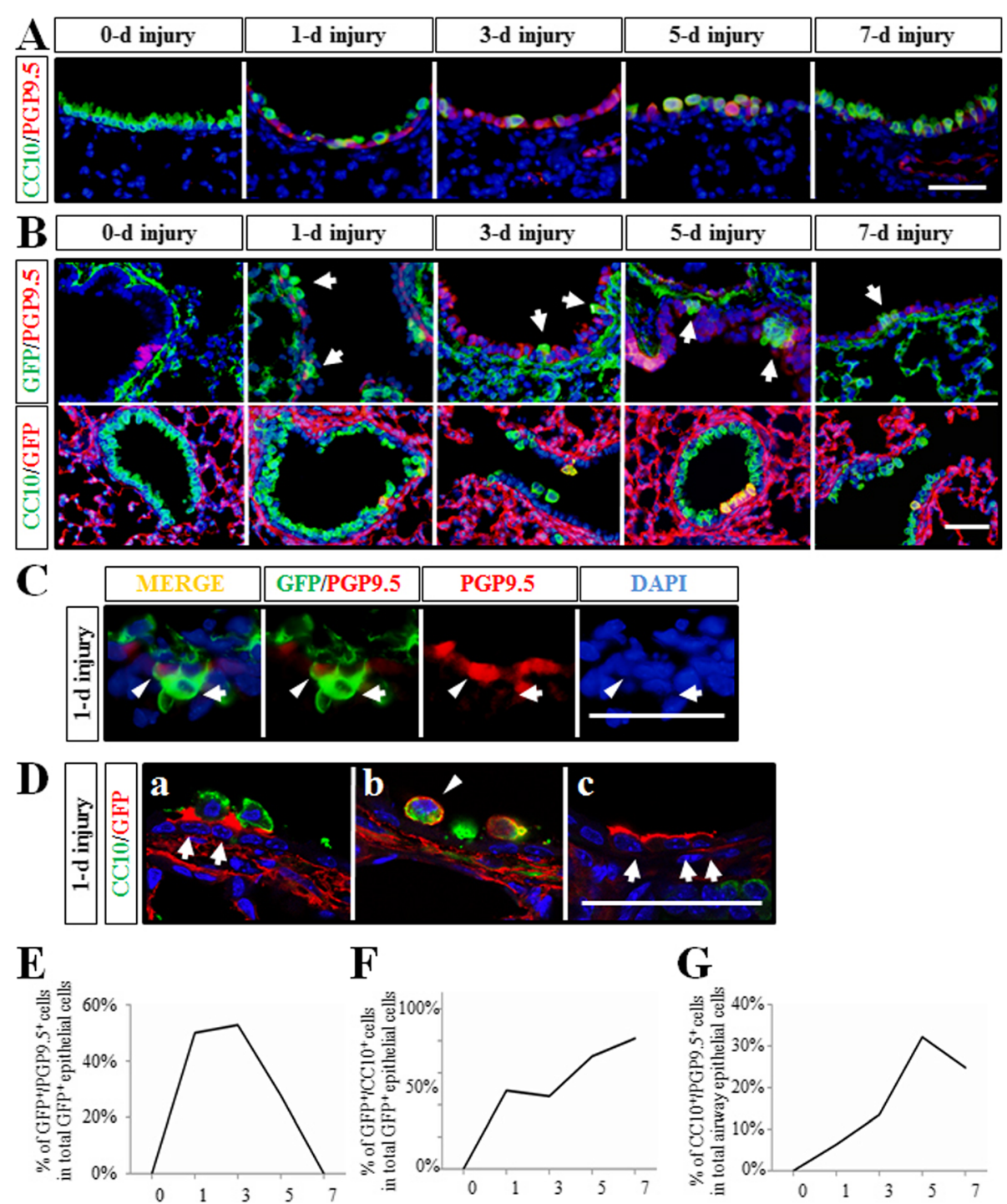

Fig. 5 The process of Dermo ${ }^{+}$stem cells in Club cell regeneration in NAPH injury repair. A Establishment of NAPH injury model. Club cells were ablated gradually at day 1 and almost restored at day 7. NE cells increased and served as stem cell during Club cell regeneration. B Dermo ${ }^{+}$cells (arrow) appeared in the airway epithelium after NAPH injury displayed as GFPpositive/PGP9. $5^{\text {positive }}$ cells (upper panel) and GFPpositive $/$ CC $10^{\text {positive }}$ cells (lower panel) respectively. C Amplified display of GFP ${ }^{+}$NE cells (arrowhead) or non-NE cells (arrow) in the airway epithelium at day 1. D Both GFPpositive $/ \mathrm{CC} 10^{\text {positive }}$ cells (arrowhead) and non-Club cells (arrow) in the airway epithelium were detected at day 1. E Dynamic changes of the

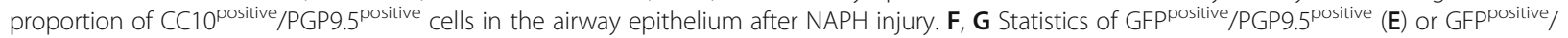
$\mathrm{CC}_{10}{ }^{\text {positive }}(\mathbf{F})$ cells over total GFPpositive Dermol stem cells after NAPH injury. d, days post NAPH injury. Scale bar $=50 \mu \mathrm{m}$

exposed to LPS. Expression of CD44 and CD90 was found in very few Dermo $1^{+}$cells throughout the airways at day 3 after LPS exposure (Fig. 7a).

To address whether the Dermo $1^{+}$epithelial cells occurred in injury repair maintained characteristic of mesenchymal cells, the expression of pulmonary mesenchymal cell markers, PDGFA, DESMIN, $\alpha$ SMA, and SMMHC, were analyzed in Dermo $^{+}$epithelial cells. In multiple experiments, we detected no expression of any of these genes in Dermo1 ${ }^{+}$epithelial cells (Fig. 7b).

\section{Discussion}

The purpose of the current study was to determine the precise role of endogenous mesenchymal stem cells in lung injury repair. The choice of Dermo1 was based on its specific expression in the pulmonary mesenchyme. In Dermo1-Cre; ROSA ${ }^{m T m G}$ mice, Dermo1 ${ }^{+}$cells were lineage traced by GFP labeling. Examination of airway epithelial regeneration in the LPS- and NAPH-induced injury/repair model revealed a process of Dermo1 ${ }^{+}$cell population dynamics characterized by expression of 

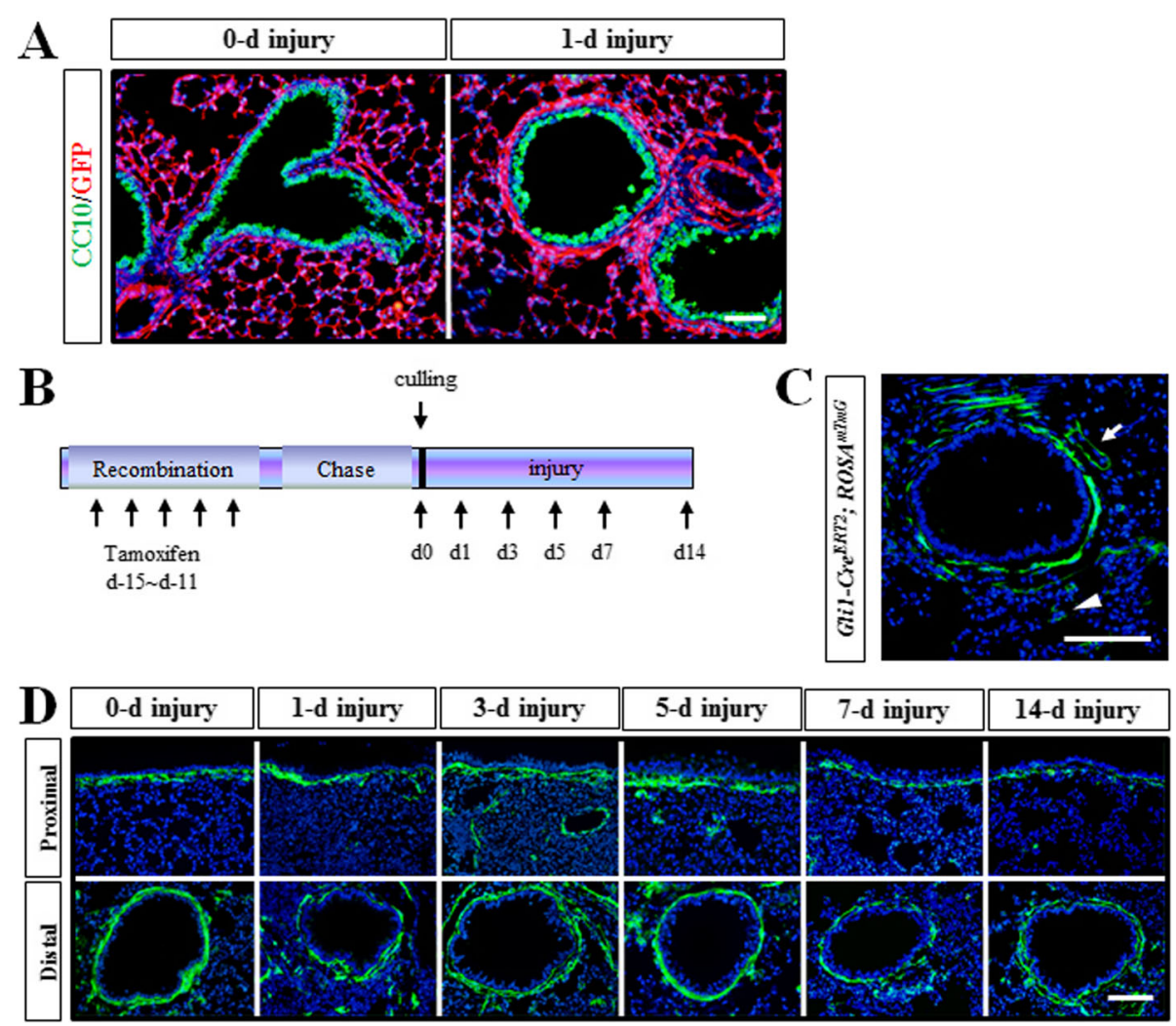

Fig. $6 \mathrm{Dermo}^{+}$stem cells were not Gli1 signaling related. a After NAPH injury, the pulmonary sub-epithelial mesenchymal layer was thickened in Dermo 1-Cre; ROSA ${ }^{m T m G}$ mice. b Timeline of lineage tracing of GliT-Cre ${ }^{E R T 2} ; R O S A^{m T m G}$-labeled cells with tamoxifen treatment in NAPH injury repair. GliT-Cre ${ }^{E R T 2} ; R O S A^{m T m G}$ mice received five continuous dose of tamoxifen via intraperitoneal injection. Single dose of NAPH injury was conducted following 10 days of chasing. Lung tissues were collected at days 1, 3, 5, or 7. c Immunofluorescence staining results showed that GFP-labeled cells were mainly in the sub-epithelial and the peri-vascular (arrow) mesenchymal area in Gli1-Cre ${ }^{E R T 2} ;$ ROSA ${ }^{\text {mTmG }}$ mice. A few of GFP ${ }^{+}$cells were also detected in the sub-meshothelial mesenchyme (arrowhead). $\mathbf{d}$ Gli $1^{+}$cells were detected in airway epithelium after LPS injury. d, days post NAPH injury. Scale bar $50 \mu \mathrm{m}$

epithelial cell markers that lead to regeneration of Club cells, ciliated cells, and goblet cells. During this wellorchestrated process, Dermo1 ${ }^{+}$mesenchymal stem cells (MSCs) sequentially transdifferentiate into epithelial stem cells and terminal differentiated epithelial cells. These studies provide novel evidence that a subgroup of Dermo $1^{+}$cells serves as endogenous pulmonary MSCs in the re-establishment of a functional airway epithelium by regulating the step-wise transition of putative stem cells to transitional epithelial stem cell intermediates and, finally, to newly differentiated epithelial cells.

Lung mesenchyme is a critical determinant of the shape and size of the lung, the extent and patterning of epithelial branching, and the formation of the pulmonary vasculature and interstitial mesenchymal components of the adult lung [21]. Compared with epithelium, the composition of the pulmonary mesenchyme is more complicated. Crosstalk between pulmonary mesenchyme and epithelium has been demonstrated during lung development and injury repair. Different mesenchymal progenitor/stem cells have been identified to provide the niches for epithelia to maintain their stem cell capacity. For example, Axin $2^{+}$Pdgfra ${ }^{+}$mesenchymal cells serve as alveolar stem cell niche supporting alveolar cell growth and regeneration, and Axin $2^{+}$Pdgfra ${ }^{-}$myofibrogenic progenitors contribute to pathologically deleterious myofibroblasts [22]. Lgr6 ${ }^{+}$mesenchymal cells are also found to promote epithelial progenitors differentiate into airway cells via Wnt-Fgf10 cooperation, whereas $\mathrm{Lgr}^{+}$cells promote epithelial progenitors differentiate into alveolar cells through Wnt pathway [23]. Meanwhile, whether MSCs can differentiate into epithelial cells in injury repair is wildly studied. BMMSCs have shown the capability to ameliorate lung injury and generated a significant amount of interests as a potential therapy for acute or chronic lung diseases [24, 25]. MSCs work through multiple mechanisms, including cell engraftment, immunomodulation, alveolar fluid clearance, lung protein permeability, and antibacterial properties [26]. However, a number of issues central to whether or not using stem cells in therapeutic approaches will be successful. Problems with interpreting the results of these studies 


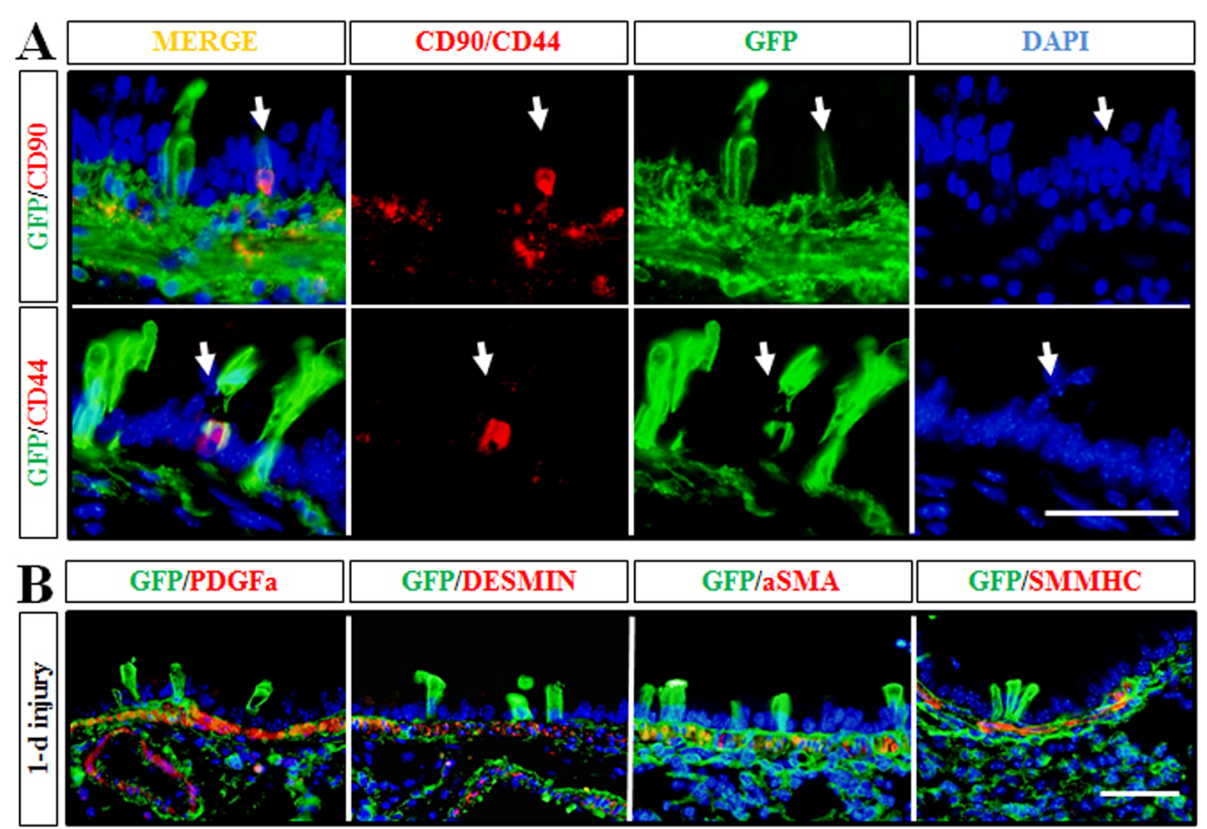

Fig. 7 Analysis of Dermo $1^{+}$stem cells. a Very few Dermo $1^{+}$lineage stem cells in the airway epithelium were positive stained for mesenchymal stem cell markers, CD90 and CD44, after LPS injury. $\mathbf{b}$ Dermo $1^{+}$lineage stem cells in the airway epithelium were negative stained for general mesenchymal markers after LPS injury, including PDGFA, DESMIN, aSMA, and SMMHC. Scale bar $=50 \mu \mathrm{m}$

include having limited controls, failure of the stem cells to engraft, and the generation of inflammatory events by the treatment itself. In addition, the processing procedures, like isolation, in vitro amplification, immunological analysis [27], and uncontrollable change of cell morphology [24], will greatly limit their wide clinical applications. Thus, more and more tissue-resident endogenous mesenchymal stem cells are studied in a variety of tissues or organs, such as fat [28], placenta and blood [29], and skeletal muscle [30].

The lung endogenous MSCs are first reported in 2007 that plastic-adherent fibroblastoid cells isolated from bronchoalveolar lavage fluid in patients undergoing lung transplant surgery are identified as MSCs [31]. The studies related to lung endogenous MSCs are carried out, most of which are implemented in vitro. In vivo studies showed minimal MSC activities which cannot exclude the result of auto-fluorescence of immunofluorescence reaction, and the therapeutic benefit is likely triggered by a paracrine-mediated mechanism [26]. Due to the lack of cell-specific markers, the understanding of the cellular interrelationships and dynamics between MSCs and epithelial cells in airway injury repair is emerged from robust cell lineage analyses. In our current study, by lineage tracing Dermo $1^{+}$-GFP cells, we discovered that a subgroup of Dermo1 ${ }^{+}$cells served as stem cells in LPS- and NAPH-induced lung injury repair. Those endogenous mesenchymal stem cells migrated into the airway epithelium layer and transdifferentiated into epithelial stem cells, which in turn repaired the damaged epithelial cells, such as Club cells, ciliated cells and goblet cells (Fig. 8). Statistical analysis of the histological preparations in NAPH injury repair indicated that both GFP ${ }^{\text {positive }} /$ PGP9. $5^{\text {positive }}$ and $\mathrm{GFP}^{\text {positive }} / \mathrm{CC} 10^{\text {positive }}$ cells appeared on day 1 after injury, which was earlier than PGP9. $5^{\text {positive }} / \mathrm{CC} 10^{\text {positive }}$ (Fig. 5). The data suggested that Dermo1 ${ }^{+}$MSCs migrated into the airway and transdifferentiated into epithelial stem cells, such as NE cells, and in turn regenerated the damaged epithelial Club cells. Previous studies also discovered that airway basal and Club cells serve as the primary progenitors of ciliated, goblet, and alveolar epithelial cells [32, 33]. Thus, we examined whether Dermo1 ${ }^{+}$MSCs transdifferentiated into basal cells during LPS injury repair. By P63 and GFP antibody double staining, we did not find GFP Gositive/ $^{2}$ p63 ${ }^{\text {positive }}$ cells on day 1 after LPS injury (Additional file 1: Figure S1). Since the majority of GFP ${ }^{\text {posi- }}$ tive cells were $\mathrm{CC} 10^{\text {positive }}$, it is possible that Dermo $^{+}$ MSCs might differentiate into "club stem cells (or variant club cells)" during LPS injury. Meanwhile, the GFP $^{\text {positive }} /$ Foxj $1^{\text {positive }}$ and GFP Positive $_{\text {MUC5AC }}$ positive $^{\text {M }}$ population did not increase from day 3 to day 14 (data not show); the possibility of Dermo $1^{+}$MSCs-Club stem cell-ciliated or goblet cell differentiation remains low. However, due to the lack of specific markers, "Club 


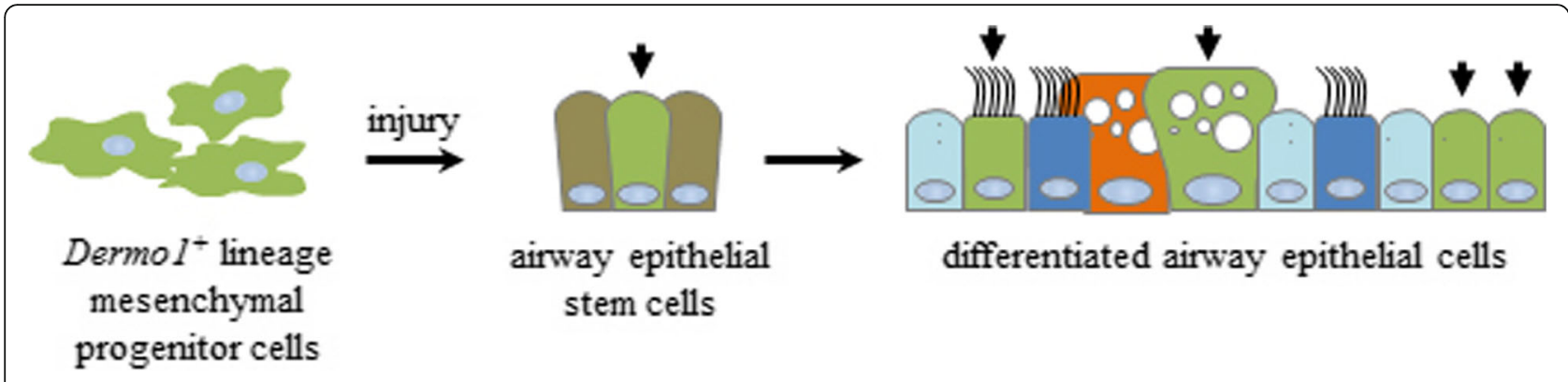

Fig. 8 Schematic diagram of the mechanisms that Dermo $1^{+}$mesenchymal progenitor cells repaired the damaged airway epithelium. Dermo ${ }^{+}$ mesenchymal stem cells firstly differentiated into epithelial stem cells and then regenerated the damaged epithelial cells via epithelial stem cells

stem cells" or other type(s) of epithelial stem cells Dermo1 $^{+}$MSCs transdifferentiated into is a query that had not been hitherto addressed.

The crosstalk between pulmonary epithelium and mesenchyme was widely reported. Among which, Shh signaling pathway played an important role. $\mathrm{Gli1}^{+}$cells traced by Shh signaling pathway reporter mice acted as progenitor cells and promoted repair of the damaged epithelial cells through paracrine actions [34]. Gli1 ${ }^{+}$cells were peri-bronchial mesenchymal cells anatomically closer to the epithelial cell layer, which were also a subgroup of Dermo1 ${ }^{+}$cells. By tracing $\mathrm{Gli1}^{+}$mesenchymal cells, surprisingly, $\mathrm{Gli1}^{+}$cells did not contribute to the regeneration of airway epithelial after LPS injury. We realized that the current data could not distinguish which signaling pathways activated the Dermo1 ${ }^{+}$MSCs. The detailed mechanisms and the factors involved in required further investigation.

Acute lung injury (ALI) is an important problem in humans; however, its pathogenesis is poorly understood. To investigate the molecular mechanisms of ALI, various experimental models have been used, the most common being the endotoxin (bacterial LPS) model. In our study, intratracheal instillation of high dose of LPS caused severe lung injury as the inflammatory reaction was still intense at 7 days after injury. Various epithelial and mesenchymal derived cells were extensively damaged. Interestingly, the emergence of Dermo1 ${ }^{+}$MSCs in the airway epithelium was found in 1 day after injury. The quick response of Dermo $1^{+}$MSCs also occurred in the NAPH-induced injury model, which was even earlier than the presence of epithelial stem cells. Based on our observations, activation of endogenous mesenchymal stem cells, such as Dermo $1^{+}$MSCs, maybe a more preferable therapeutic approach than engrafting of bone marrow-derived MSCs.

\section{Conclusion}

In sum, the results of this study suggest that, during mouse lung LPS and naphthalene injury repair, a population of Dermo1 $^{+}$mesenchymal cells serve as a reservoir for epithelial cell regeneration and re-establishment of the normal airway epithelium. The Dermo1 ${ }^{+}$mesenchymal stem cell differentiated into epithelial stem cells before reestablishing various epithelial cells. These findings have implications for understanding the regulation of lung repair and the potential for usage of mesenchymal stem cells in therapeutic strategies for lung diseases.

\section{Supplementary information}

Supplementary information accompanies this paper at https://doi.org/10. 1186/s13287-019-1452-1.

Additional file 1: Figure S1. Dermol ${ }^{+}$stem cells did not

transdifferentiated into basal cell in LPS injury repair. GFP and P63 antibody staining results showed that Dermo $1^{+}$stem cells did not labeled by P63 in airway epithelium at $6 \mathrm{~h}$ and 1 day after LPS injection. Scale bar $=50 \mu \mathrm{m}$.

Additional file 2: Figure S2. Dermo $1^{+}$stem cells were not SOX2 or SOX9 positive cells in LPS injury repair. By immunostaining, the result showed that Dermo $1^{+}$stem cells did not express Sox2 and Sox9 in airway epithelium at 1 and 3 day after LPS injection. E14.5 wild type lung was used as positive control. Scale bar $=50 \mu \mathrm{m}$.

\section{Abbreviations}

LPS: Lipopolysaccharide; MSC: Mesenchymal stem cell; NAPH: Naphthalene; NE: Neuroendocrine

\section{Authors' contributions}

YX contributed to the study concept and design, analysis and interpretation of data, drafting of the manuscript. SF and SZ contributed acquisition of data, analysis and interpretation of data. HD contributed to the acquisition of data. TT contributed to the technical and material support. XH provided the animals. CL contributed to the research design, analysis and interpretation of data. All authors read and approved the final manuscript.

\section{Funding}

This work is supported by National Basic Research Program (2016YFA0100202), the Project for Extramural Scientists of Beijing Advanced Innovation Center for Food Nutrition and Human Health, the Project for Extramural Scientists of State Key Laboratory of Agrobiotechnology (2019SKLAB6-22) and China Postdoctoral Science Foundation Funded Project (2018M641550 \& 2019T120161).

Availability of data and materials

All data generated or analyzed during this study are included in this published article.

Ethics approval and consent to participate

All experimental procedures for handling and care of mice were following the instructions of the Institutional Animal Care Committee and approved by 
the Ethics Committee of the Agriculture University of China (Permission Number:

CAU20161010-1, valid period: 10,10,2016-9,22,2019)

\section{Consent for publication}

Not applicable.

\section{Competing interests}

The authors declare that they have no competing interests.

\section{Author details}

'State Key Laboratory for Agrobiotechnology, College of Biological Sciences, China Agricultural University, Beijing, People's Republic of China. ${ }^{2}$ National Engineering Laboratory for Animal Breeding, China Agricultural University, Beijing, China. ${ }^{3}$ Department of Pediatrics, Division of Neonatology, University of Southern California, Keck School of Medicine, Los Angeles, CA, USA.

Received: 21 June 2019 Revised: 11 September 2019 Accepted: 11 October 2019 Published online: 02 December 2019

\section{References}

1. Lynch TJ, Anderson PJ, Rotti PG, Tyler SR, Crooke AK, Choi SH, et al. Submucosal gland myoepithelial cells are reserve stem cells that can regenerate mouse tracheal epithelium. Cell Stem Cell. 2018;22:653-67 e655.

2. Rock JR, Onaitis MW, Rawlins EL, Lu Y, Clark CP, Xue Y, et al. Basal cells as stem cells of the mouse trachea and human airway epithelium. Proc Natl Acad Sci U S A. 2009;106:12771-5.

3. Kim CF, Jackson EL, Woolfenden AE, Lawrence S, Babar I, Vogel S, et al. Identification of bronchioalveolar stem cells in normal lung and lung cancer. Cell. 2005;121:823-35.

4. Barkauskas CE, Cronce MJ, Rackley CR, Bowie EJ, Keene DR, Stripp BR, et al. Type 2 alveolar cells are stem cells in adult lung. J Clin Invest. 2013;123: 3025-36.

5. Metzger RJ, Klein OD, Martin GR, Krasnow MA. The branching programme of mouse lung development. Nature. 2008;453:745-50.

6. Yin Y, Wang F, Ornitz DM. Mesothelial- and epithelial-derived FGF9 have distinct functions in the regulation of lung development. Development. 2011;138:3169-77.

7. Morimoto M, Liu Z, Cheng HT, Winters N, Bader D, Kopan R. Canonical Notch signaling in the developing lung is required for determination of arterial smooth muscle cells and selection of Clara versus ciliated cell fate. J Cell Sci. 2010;123:213-24.

8. Swartz D. Some developmental characters of species of Lycoperdaceae. Am J Bot. 1933;20:440-65.

9. Sekine K, Ohuchi H, Fujiwara M, Yamasaki M, Yoshizawa T, Sato T, et al. Fgf10 is essential for limb and lung formation. Nat Genet. 1999;21:138-41.

10. Xing Y, Li A, Borok Z, Li C, Minoo P. NOTCH1 is required for regeneration of Clara cells during repair of airway injury. Stem Cells. 2012;30:946-55.

11. Rittirsch D, Flierl MA, Day DE, Nadeau BA, McGuire SR, Hoesel LM, et al. Acute lung injury induced by lipopolysaccharide is independent of complement activation. J Immunol. 2008;180:7664-72.

12. Arnhold $\mathrm{S}$, Heiduschka $\mathrm{P}$, Klein $\mathrm{H}$, Absenger $\mathrm{Y}$, Basnaoglu $\mathrm{S}$, Kreppel F, et al. Adenovirally transduced bone marrow stromal cells differentiate into pigment epithelial cells and induce rescue effects in RCS rats. Invest Ophthalmol Vis Sci. 2006;47:4121-9.

13. Gupta N, Su X, Popov B, Lee JW, Serikov V, Matthay MA. Intrapulmonary delivery of bone marrow-derived mesenchymal stem cells improves survival and attenuates endotoxin-induced acute lung injury in mice. J Immunol. 2007;179:1855-63.

14. Spees JL, Pociask DA, Sullivan DE, Whitney MJ, Lasky JA, Prockop DJ, et al. Engraftment of bone marrow progenitor cells in a rat model of asbestosinduced pulmonary fibrosis. Am J Respir Crit Care Med. 2007;176:385-94.

15. Buisine MP, Devisme L, Copin MC, Durand-Reville M, Gosselin B, Aubert JP, et al. Developmental mucin gene expression in the human respiratory tract. Am J Respir Cell Mol Biol. 1999;20:209-18.

16. Locksley RM, Killeen N, Lenardo MJ. The TNF and TNF receptor superfamilies: integrating mammalian biology. Cell. 2001;104:487-501.

17. Tanaka T, Narazaki M, Kishimoto T. IL-6 in inflammation, immunity, and disease. Cold Spring Harb Perspect Biol. 2014;6:a016295.
18. de Waal MR, Abrams J, Bennett B, Figdor CG, de Vries JE. Interleukin 10(IL10) inhibits cytokine synthesis by human monocytes: an autoregulatory role of IL-10 produced by monocytes. J Exp Med. 1991;174:1209-20.

19. Yu K, Xu J, Liu Z, Sosic D, Shao J, Olson EN, et al. Conditional inactivation of FGF receptor 2 reveals an essential role for FGF signaling in the regulation of osteoblast function and bone growth. Development. 2003;130:3063-74.

20. Li M, Li C, Liu YH, Xing Y, Hu L, Borok Z, et al. Mesodermal deletion of transforming growth factor-beta receptor II disrupts lung epithelial morphogenesis: cross-talk between TGF-beta and Sonic hedgehog pathways. J Biol Chem. 2008;283:36257-64.

21. Yin Y, White AC, Huh SH, Hilton MJ, Kanazawa H, Long F, et al. An FGF-WNT gene regulatory network controls lung mesenchyme development. Dev Biol. 2008;319:426-36.

22. Zepp JA, Zacharias WJ, Frank DB, Cavanaugh CA, Zhou S, Morley MP, et al. Distinct mesenchymal lineages and niches promote epithelial self-renewal and myofibrogenesis in the lung. Cell. 2017;170:1134-48 e1110.

23. Lee JH, Tammela T, Hofree M, Choi J, Marjanovic ND, Han S, et al. Anatomically and functionally distinct lung mesenchymal populations marked by Lgr5 and Lgr6. Cell. 2017;170:1149-63 e1112.

24. Kumamoto M, Nishiwaki T, Matsuo N, Kimura H, Matsushima K. Minimally cultured bone marrow mesenchymal stem cells ameliorate fibrotic lung injury. Eur Respir J. 2009:34:740-8.

25. Sun J, Han ZB, Liao W, Yang SG, Yang Z, Yu J, et al. Intrapulmonary delivery of human umbilical cord mesenchymal stem cells attenuates acute lung injury by expanding CD4+CD25+ Forkhead Boxp3 (FOXP3)+ regulatory T cells and balancing anti- and pro-inflammatory factors. Cell Physiol Biochem. 2011;27:587-96.

26. Lee JW, Fang X, Krasnodembskaya A, Howard JP, Matthay MA. Concise review: mesenchymal stem cells for acute lung injury: role of paracrine soluble factors. Stem Cells. 2011;29:913-9.

27. Krampera M, Galipeau J, Shi Y, Tarte K, Sensebe L. Therapy MSCCotISfC immunological characterization of multipotent mesenchymal stromal cells-the International Society for Cellular Therapy (ISCT) working proposal. Cytotherapy. 2013;15:1054-61.

28. Zuk PA, Zhu M, Mizuno H, Huang J, Futrell JW, Katz AJ, et al. Multilineage cells from human adipose tissue: implications for cell-based therapies. Tissue Eng. 2001;7:211-28.

29. Zvaifler NJ, Marinova-Mutafchieva L, Adams G, Edwards CJ, Moss J, Burger $J A$, et al. Mesenchymal precursor cells in the blood of normal individuals. Arthritis Res. 2000;2:477-88.

30. Jankowski RJ, Deasy BM, Huard J. Muscle-derived stem cells. Gene Ther. 2002;9:642-7.

31. Lama VN, Smith L, Badri L, Flint A, Andrei AC, Murray S, et al. Evidence for tissue-resident mesenchymal stem cells in human adult lung from studies of transplanted allografts. J Clin Invest. 2007;117:989-96.

32. Whitsett JA. Airway epithelial differentiation and mucociliary clearance. Ann Am Thorac Soc. 2018;15(Suppl 3):S143-8.

33. Zheng D, Soh BS, Yin L, Hu G, Chen $\mathrm{Q}$, Choi $\mathrm{H}$, et al. Differentiation of Club cells to alveolar epithelial cells in vitro. Sci Rep. 2017;27(7):41661.

34. Peng T, Frank DB, Kadzik RS, Morley MP, Rathi KS, Wang T, et al. Hedgehog actively maintains adult lung quiescence and regulates repair and regeneration. Nature. 2015;526:578-82.

\section{Publisher's Note}

Springer Nature remains neutral with regard to jurisdictional claims in published maps and institutional affiliations.

\section{Ready to submit your research? Choose BMC and benefit from:}

- fast, convenient online submission

- thorough peer review by experienced researchers in your field

- rapid publication on acceptance

- support for research data, including large and complex data types

- gold Open Access which fosters wider collaboration and increased citations

- maximum visibility for your research: over $100 \mathrm{M}$ website views per year

At BMC, research is always in progress.

Learn more biomedcentral.com/submissions 